\title{
Spin Hall Effect induced by resonant scattering on impurities in metals
}

\author{
Albert Fert \\ Unité Mixte de Physique CNRS/Thales, 91767, Palaiseau, \\ France and Université Paris-Sud, 91405, Orsay, France \\ Peter M Levy \\ Department of Physics, New York University, \\ 4 Washington Place, New York, NY 10003
}

(Date textdate)

\begin{abstract}
The Spin Hall Effect (SHE) is a promising way for transforming charge currents into spin currents in spintronic devices. Large values of the Spin Hall Angle, the characteristic parameter of the yield of this transformation, have been recently found in noble metals doped with nonmagnetic impurities. We show that this can be explained by resonant scattering off impurity states split by the spin-orbit interaction. We apply our calculation to the interpretation of experiments on copper doped with 5d impurities and we describe the conditions to obtain the largest effects.
\end{abstract}


The Spin Hall Effect (SHE), first described by Dyakonov and Perel in 1971[1], is a subject of intense research as it allows for the generation of spin currents in nonmagnetic conductors and the developments of spintronic devices built without ferromagnetic materials. The SHE is due to spin-orbit (S-O) interactions which deflect the spin up and spin down electrons of an electrical current in opposite directions. While the symmetry between spin up and spin down in a nonmagnetic material precludes charge accumulation on the edges of the conductor, i.e. no Hall voltage, there is a spin accumulation which can be exploited to generate a pure spin current. Alternatively a Hall voltage can be generated by injecting a spin-polarized current to break the symmetry, which is called the Inverse SHE effect. The SHE is associated with off-diagonal terms of the resistivity tensor having opposite signs for spin up and spin down electrons, respectively $\rho_{x y}$ and $-\rho_{x y}$ for $s_{z}= \pm 1 / 2$. It can include an intrinsic contribution due to the effect of S-O interactions on the wave functions of the pure material [2, 3] and an extrinsic one resulting from spin-orbit interactions on impurity or defect sites [4, 5]. Two mechanisms can contribute to the extrinsic SHE, the skew scattering [4] and the scattering with side-jump [5].

When the SHE is used to produce a transverse spin current, the maximum yield of the transformation of a longitudinal charge current into a transverse spin current is related to the Spin Hall Angle (SHA), defined as $\Phi_{H}=\rho_{x y} / \rho_{x x}$ where $\rho_{x x}$ is the diagonal term of the resistivity tensor, i.e., the conventional resistivity for spin $\sigma=\uparrow \downarrow( \pm)$ channels. Consequently $\Phi_{H}$ is the important parameter for practical applications in spintronics. Until 2007 the largest values of $\Phi_{H}$ obtained for pure materials, metals or semiconductors, had been obtained for $\operatorname{Pt}\left(\Phi_{H} \approx 0.5 \%\right)$ [6, 77. The much larger value of $5 \%$ found in 2008 for $\mathrm{Au}$ [8] was surprising and has been ascribed to skew scattering by Fe or Pt impurities [9]. An even larger SHE $(\approx 15 \%)$ was recently obtained by doping Au with Pt impurities [10. Actually this brings to mind the large values of $\Phi_{H}$ of a few percent found thirty years ago [11, 12] for the SHE induced by nonmagnetic $5 \mathrm{~d}$ impurities in $\mathrm{Cu}$, e.g. $\Phi_{H}=2.6 \%$, for $\mathrm{Cu}$ doped with Ir. This large SHE, with a typical change of sign between the beginning (Lu) and the end (Ir) of the $5 \mathrm{~d}$ series, was ascribed to resonant scattering on the impurity $5 \mathrm{~d}$ states split by the S-O interaction [11]. Recently measurements by Niimi et al [13] on Cu doped with Ir have confirmed the large value $\left(\Phi_{H} \approx 1.5 \%\right)$ of the SHE induced by Ir in $\mathrm{Cu}$ and confirmed its skew scattering mechanism. Thus, impurity scattering appears as a promising way to obtain the most efficient transformation of charge currents into spin currents by SHE. This 
has triggered the development of theoretical models of the SHE induced by impurities [14].

In this paper we present a calculation and discussion of the SHE induced by resonant scattering from impurity levels. For $5 \mathrm{~d}$ impurities in $\mathrm{Cu}$, we can explain the order of magnitude of the large SHE of the experiments and the change of sign between the beginning and the end of the $5 \mathrm{~d}$ series. Whereas the recent papers of Gradhand et al. [14] present calculations of only the skew scattering contribution, we calculate both the skew scattering and side jump terms. By comparing the spin Hall angles due to skew scattering and to side-jump, we can predict the threshold concentration at which the side-jump contribution becomes predominant and can generate very large effects. In contrast to the ab-initio calculations of Ref. [14], our calculation is performed in an analytical model which aims at a general description of the main features of the impurity-induced SHE and at a prediction of the best conditions for large effects.

Our calculation is based on a partial wave analysis of the resonant scattering of free electrons from the $j=5 / 2$ and $j=3 / 2$ states of $5 d$ impurities in a metal like $\mathrm{Cu}$, as illustrated in the inset of Fig.1. From the splitting between the $5 / 2$ and $3 / 2$ levels, $E_{5 / 2}-$ $E_{3 / 2}=5 \lambda_{d} / 2$, where $\lambda_{d}$ is the impurity $5 d$ S-O constant and by using the classical expression of the phase shift at energy $E$ as a function of the resonant level energy $E_{j}, \operatorname{ctn}\left(\eta_{j}\right)=$ $\left(E_{j}-E\right) / \Delta$ where $\Delta$ is the resonance width, we find to first order in $\lambda d / \Delta, \Delta \eta=\eta_{3 / 2}-\eta_{5 / 2}=$ $5 / 2 \frac{\lambda_{d}}{\Delta} \sin ^{2} \eta_{2}$ where $\eta_{2}$ is the mean phase shift expressed as a function of the number $Z_{d}$ of $5 d$ electrons on the impurity by Friedel's sum rule, $\eta_{2}=\left(3 \eta_{3 / 2}+2 \eta_{5 / 2}\right) / 5=\pi Z_{d} / 10$ [15]. After expanding the states $\left|j, m_{j}\right\rangle$ in terms of $|m, \sigma\rangle$ states and keeping only terms that will contribute to $\rho_{x x}$ and $\rho_{x y}$,we find the following expression for the scattering $T$-matrix (to first order in $\lambda_{d} / \Delta$ only non-spin-flip terms contribute),

$$
T_{\mathbf{k}^{\prime} \sigma, \mathbf{k} \sigma}=\frac{2}{n\left(\varepsilon_{F}\right)}\left[\sigma \frac{\lambda_{d}}{\Delta} e^{i 2 \eta_{2}} \sin ^{2} \eta_{2} \sum_{m} m Y_{2}^{m *}(\hat{k}) Y_{2}^{m}\left(\hat{k}^{\prime}\right)-2 \sum_{l m} e^{i \eta_{l}} \sin \eta_{l} Y_{l}(\hat{k}) \cdot Y_{l}\left(\hat{k}^{\prime}\right)\right]
$$

where $\sigma= \pm 1$ and $n\left(\varepsilon_{k \sigma}\right)$ is the DOS for one direction of the spin. Note that interchanging $\hat{k}$ and $\hat{k}^{\prime}$ in the first term in the bracket changes its sign; this is the signature of the antisymmetric scattering. The second term is the usual symmetric term associated with charge scattering.

From the antisymmetric part of the scattering probability $W_{\text {antisym }}\left(k \sigma \rightarrow k^{\prime} \sigma\right)$, associated with cross terms between the antisymmetric and symmetric parts of the $T$-matrix, we define 
$\omega_{\text {skew }}\left(k_{F} \sigma\right)$ by

$$
\sum_{k^{\prime}} W_{\text {antisym }}\left(\mathbf{k} \sigma \rightarrow \mathbf{k}^{\prime} \sigma\right) g\left(\mathbf{k}^{\prime}, \sigma\right) \equiv e \omega_{\text {skew }}\left(k_{F} \sigma\right) \hat{\mathbf{e}} \cdot \hat{\mathbf{k}} \times \hat{\mathbf{z}}
$$

where $\hat{\mathbf{e}}$ is a unit vectors along the electric field $\mathbf{E}, \hat{\mathbf{z}}$ the spin quantization axis, $\tau_{0}$ the isotropic relaxation time and we have used the normal out-of-equilibrium distribution function $g\left(\mathbf{k}^{\prime}, \sigma\right) \equiv-e \tau_{0} v_{F} \hat{\mathbf{e}} \cdot \hat{\mathbf{k}}^{\prime}$ to arrive at this result. From the cross terms in the $T$-matrix between the $l=2$ and $l \pm 1$ terms and upon performing the integrals over spherical conduction bands we find

$$
\omega_{\text {skew }}\left(k_{F} \sigma\right)=-\sigma \frac{6 N_{i} \tau_{0} v_{F}}{\pi \hbar n\left(\varepsilon_{F}\right)} \frac{\lambda_{d}}{\Delta} \sin \left(2 \eta_{2}-\eta_{1}\right) \sin ^{2} \eta_{2} \sin \eta_{1}
$$

where $N_{i}$ is the number of impurities.

The side jump contribution to the SHE enters when we write Hall current in the presence of spin-orbit scattering [16] as $\mathbf{J}=-e \sum_{\mathbf{k}, \sigma}\left[\mathbf{v}_{\mathbf{k}}+\omega_{a}(\mathbf{k}, \sigma] f(\mathbf{k}, \sigma)\right.$ where the term $\omega_{a}$ is the anomalous velocity [arising from a side jump] attendant to electron flow in systems with spin-orbit coupling [17]. By using the distribution function $f(\mathbf{k}, \sigma)$ found from the linearized Boltzmann equation that accounts for antisymmetric (see Eq. 2) as well as symmetric scattering and the appearance of side jumps [18], we find the current can be written as

$$
\begin{aligned}
\mathbf{J}(\sigma & \left.=\uparrow \downarrow)=e^{2} E \sum_{\mathbf{k}}\left(-\frac{\partial f^{0}}{\partial \varepsilon_{k \sigma}}\right) \tau_{0}\left(k_{F} \sigma\right)\left[v_{F} \hat{\mathbf{k}}-\omega_{a}(k, \sigma) \hat{\mathbf{z}} \times \hat{\mathbf{k}}\right)\right] \\
& \left.\times \hat{\mathbf{e}} \cdot\left[v_{F} \hat{\mathbf{k}}+\left\{\omega_{\text {skew }}\left(k_{F}, \sigma\right)+\omega_{a}\left(k_{F}, \sigma\right)\right\}(\hat{\mathbf{z}} \times \hat{\mathbf{k}})\right)\right]
\end{aligned}
$$

where we have written $\omega_{a}(\mathbf{k}, \sigma)=\omega_{a}(\mathbf{k}, \sigma) \hat{k} \times \hat{z}$.

The transverse Hall current comes from terms proportional to $\hat{\mathbf{z}} \times \hat{\mathbf{e}}$. When we consider spherical conduction bands and average over $\Omega_{k}$ we find there are two transverse components in $\sigma_{H} \equiv \sigma_{y x}$. The skew scattering one is

$$
\sigma_{\text {skew }}(\sigma=\uparrow \downarrow)=-\frac{1}{3} e^{2}\left[\int n\left(\varepsilon_{k \sigma}\right) d \varepsilon_{k \sigma}\left(-\frac{\partial f^{0}}{\partial \varepsilon_{k \sigma}}\right) v\left(\varepsilon_{k \sigma}\right) \tau_{0}\left(k_{F} \sigma\right) \omega_{\text {skew }}\left(k_{F}, \sigma\right)\right],
$$

and the anomalous velocity or side jump contribution is,

$$
\sigma_{\text {anom }}(\sigma=\uparrow \downarrow)=-\frac{2}{3} e^{2}\left[\int n\left(\varepsilon_{k \sigma}\right) d \varepsilon_{k \sigma}\left(-\frac{\partial f^{0}}{\partial \varepsilon_{k \sigma}}\right) v\left(\varepsilon_{k \sigma}\right) \tau_{0}\left(k_{F} \sigma\right) \omega_{\mathbf{a}}\left(k_{F}, \sigma\right)\right] .
$$




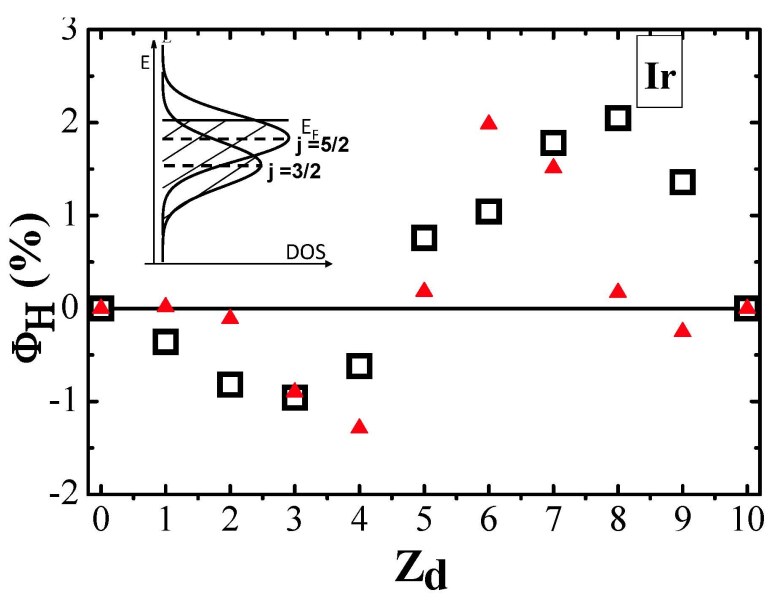

FIG. 1: Skew scattering (squares) and side-jump (triangles) contributions to the Spin Hall Angle calculated from Eqs.9 and 13 as a function of the number of d electrons, $Z_{d}$, for $5 d$ impurities in $C u$. The parameters are indicated in the text. The side-jump contribution is calculated for an impurity concentration of $2 \%$. Inset: Density of States (DOS) of a $5 d$ virtual bound state with S-O splitting between $j=3 / 2$ and $j=5 / 2$ states.

The normal conductivity for each spin channel is

$$
\sigma_{N}^{-1}=\frac{20 \pi \hbar N_{i}}{n_{\sigma} e^{2} k_{F}} \sin ^{2} \eta_{2}
$$

where $k_{F}$ is the momentum at the Femi level, and $n_{\sigma}=\frac{1}{2} n_{\text {total }}$. By placing the expression for $\omega_{\text {skew }}\left(k_{F} \sigma\right)$, see Eq. 3, in the expression for $\sigma_{\text {skew }}$, Eq. 5, and dividing by $\sigma_{N}^{2}$ we find to first order in $\sigma_{H} / \sigma_{N}$ the skew scattering contribution to the Hall effect is,

$$
\rho_{x y}^{\text {skew }}(\sigma=\uparrow \downarrow \rightarrow \pm)= \pm \frac{12 \pi N_{i} \hbar}{n_{\sigma} e^{2} k_{F}} \frac{\lambda_{d}}{\Delta} \sin \left(2 \eta_{2}-\eta_{1}\right) \sin ^{2} \eta_{2} \sin \eta_{1} .
$$

The Hall angle from the skew scattering is

$$
\Phi_{H}^{\text {skew }}= \pm 3 / 5 \frac{\lambda_{d}}{\Delta} \sin \left(2 \eta_{2}-\eta_{1}\right) \sin \eta_{1}
$$

The contribution to the Hall effect from the side jump mechanism, i.e., the anomalous velocity, is found by repeating the calculation done for the Kondo-like rare-earth ions [16], but this time using the $T$ matrix for resonant $d$ states, Eq.1. The expression for the anomalous velocity $\omega_{a}(\mathbf{k}, \sigma)$,Eq. (2.13) in Ref.16 is, 


$$
\begin{aligned}
\omega_{a}(\mathbf{k}, \sigma) & =\frac{2 N_{i}}{\hbar}\left[\operatorname{Re} \nabla_{\mathbf{k}} T_{\mathbf{k} \sigma, \mathbf{k} \sigma}+\sum_{\mathbf{k} / \sigma^{\prime}} P \frac{1}{\left(\varepsilon_{k \sigma}-\varepsilon_{k^{\prime} \sigma^{\prime}}\right)} \operatorname{Re} T_{\mathbf{k} \sigma, \mathbf{k}^{\prime} \sigma^{\prime}}^{\dagger} \nabla_{\mathbf{k}^{\prime}} T_{\mathbf{k}^{\prime} \sigma^{\prime}, \mathbf{k} \sigma}\right. \\
& \left.-\pi \sum_{\mathbf{k} / \sigma^{\prime}} \delta\left(\varepsilon_{k \sigma}-\varepsilon_{k^{\prime} \sigma^{\prime}}\right) \operatorname{Im} T_{\mathbf{k} \sigma, \mathbf{k}^{\prime} \sigma^{\prime}}^{\dagger} \nabla_{\mathbf{k}^{\prime}} T_{\mathbf{k}^{\prime} \sigma^{\prime}, \mathbf{k} \sigma}\right]
\end{aligned}
$$

Only the last term contributes to the Hall effect [16] and by using Eq. 1, we find

$$
\omega_{a}(\mathbf{k}, \sigma)=\sigma \frac{12 N_{i}}{\pi n\left(\varepsilon_{F}\right) \hbar k_{F}} \frac{\lambda_{d}}{\Delta} \frac{E_{F}}{\Delta} \cos \left(3 \eta_{2}-\eta_{1}\right) \sin ^{3} \eta_{2} \sin \eta_{1} \hat{\mathbf{k}} \times \hat{\mathbf{z}} .
$$

By placing this expression in Eq. 6, and dividing by $\sigma_{N}^{2}$ we find the anomalous velocity contribution to the Hall effect is,

$$
\rho_{x y}^{\text {anom }}(\sigma=\uparrow \downarrow \rightarrow \pm)=\mp \frac{320 N_{i} \hbar}{n_{\sigma} e^{2} k_{F}} \frac{c}{z} \frac{\lambda_{d}}{\Delta} \frac{E_{F}}{\Delta} \cos \left(3 \eta_{2}-\eta_{1}\right) \sin ^{5} \eta_{2} \sin \eta_{1}
$$

where $c$ is the impurity concentration, and $z \equiv \frac{n_{\text {total }}}{N_{s}}=\frac{2 n_{\sigma}}{N_{s}}$, i.e., the number of conduction electrons per lattice site. Finally, the Hall angle from the side jump is,

$$
\Phi_{H}^{\text {anom }}=\mp 16 / \pi \frac{c}{z} \frac{\lambda_{d}}{\Delta} \frac{E_{F}}{\Delta} \cos \left(3 \eta_{2}-\eta_{1}\right) \sin ^{3} \eta_{2} \sin \eta_{1} .
$$

Similar calculations can be performed in the presence of crystal field. With completely crystal field split $t_{2 g}$ and $e_{g}$ states, for example, the prefactors of Eqs. (9) and (13) for the $t_{2 g}$ states are multiplied by $\frac{1}{3}$ and $\frac{1}{5}$ respectively, and $\eta_{2}$ is replaced by $\eta_{t_{2 g}}=\frac{\pi}{6} Z_{t_{2 g}}$, where $Z_{t_{2 g}}$ is the number of electrons in the $t_{2 g}$ states.

We begin the discussion of our results by a glance at the expressions of the Spin Hall Angle (SHA) for skew scattering and side-jump, respectively Eqs. (9) and (13), for the case without crystal field splittings. $\Phi_{H}^{\text {skew }}$ is proportional to $\frac{\lambda_{d}}{\Delta}$, and $\Phi_{H}^{\text {anom }} \sim \frac{\lambda_{d} E_{F}}{\Delta^{2}}$. Large effects are thus expected for narrow resonances when the $\mathrm{S}-\mathrm{O}$ splitting induces significant differences in the scattering on $5 / 2$ and $3 / 2$ states. In the corresponding expressions for the intrinsic contribution to the SHA [2, 3] the denominator $\Delta$ is replaced by an energy of the order of the band width, therefore extrinsic effects due to resonant scattering should be generally larger in the usual case where the width of the resonance is smaller than the band width.

The second important feature in the expressions for the Hall angle, arising from the symmetry rules for the SHE, is the interplay between the asymmetric scattering amplitude 
in the channel $l$ and the symmetric amplitudes in the channels $l \pm 1$. It follows that the Spin Hall angle, Eqs. (9) and (13), depends not only on the phase shift $\eta_{2}$ in the resonant channel $(l=2)$ but also on the phase shift $\eta_{1}$ in the non-resonant channel $l=1$ (we have neglected the phase shift in the channel with $l=3$ ). As the scattering in a nonresonant channel is generally weaker than in a resonant one, this selection of cross terms between different spherical harmonics (rarely described in theoretical papers) contributes to the general smallness of the SHA.

We now focus on the skew scattering. If one supposes, as generally admitted, that the main contribution to the scattering by $5 d$ impurities in noble metals comes from the resonance on their $5 \mathrm{~d}$ states [15], $\eta_{2}$ is much larger then $\eta_{1}$ and, in first approximation, $\Phi_{H}$ is proportional to $\sin 2 \eta_{2}$; see Eq. (9). As $\eta_{2}=\frac{\pi Z_{d}}{10}, \sin 2 \eta_{2}$ changes sign from positive to negative between the beginning and end of the $5 \mathrm{~d}$ series as shown in Fig.1. This change arises from the difference in sign of the asymmetric resonant scattering on 5/2 and 3/2 states. This agrees with the observed change of sign for the skew scattering SHE induced by $5 \mathrm{~d}$ impurities in $\mathrm{Cu}$ [11, 12], $\Phi_{H}=-1.2 \%$ for Lu impurities $\left(Z_{d}[L u]=1\right)$ and $\Phi_{H}=2.6 \%$ for Ir impurities $\left(Z_{d}[I r]=8\right)$; the positive SHE for Ir in $\mathrm{Cu}$ has been confirmed by recent experiments [13]. A similar change in sign with $Z_{d}$ is observed also for pure 5 d metals, which suggests a similar explanation based on the relative position of the $5 d$ states, split by the S-O interaction, with respect to the Fermi level. However,the crystal field splitting should also be taken into account for a precise prediction of the variation in the $5 d$ series. As we have summarized after Eq. 13 the crystal field splitting between $t_{2 g}$ and $e_{g}$ leads to a variation of the SHE as $\sin \frac{\pi Z_{t_{2 g}}}{3}$ with a change of sign for $Z_{t_{2 g}}=3$.

Now, we proceed to a quantitative discussion of the skew scattering Hall angle predicted by Eq. (9). First we discuss $C u$ doped with $I r$ for which different types of experiments have shown a predominant contribution from skew scattering with reasonably consistent values of $\Phi_{H}, \Phi_{H}=2.6 \%$ in Ref.[11] and $\Phi_{H}=1.5 \%$ in Ref.[13] . Typical values of $\Delta$ for $5 d$ impurities in noble metals are close to $0.5 \mathrm{eV}$ from both experiments [19] and ab-initio calculations [9, 20]. With $\Delta=0.5 \mathrm{eV}, \lambda_{d} \approx 0.25 \mathrm{eV}$ [21], and $Z_{d}=8$, the mean experimental value of the SHA for CuIr, $\Phi_{H}=2.05 \%$, is obtained by introducing $\eta_{1}=-4.3^{\circ}$ in Eq. (9). The decomposition of Eq. (9) into two factors, $\frac{3 \lambda_{d} \sin \left(2 \eta_{2}-\eta_{1}\right)}{5 \Delta}=-0.277$ and $\sin \eta_{1}=-0.075$, shows that the interference between the resonant and nonresonant channels induces a significant reduction. The calculation for $I r$ in $C u$ can be extended to other 5 d impurities. With the 
same values of $\Delta, \eta_{1}$, and using the S-0 constants $\lambda_{d}$ for the $5 \mathrm{~d}$ series [21] and $\eta_{2}=\frac{\pi Z_{d}}{10}$, one obtains the wavy variation of $\Phi_{H}$ as a function of $Z_{d}$ shown in Fig.1.

In contrast to the skew scattering contribution to the SHA, the side jump one is proportional to the impurity concentration $c$. The side-jump SHA for $c=2 \%$, calculated with $E_{F}=7 \mathrm{eV}$ for $\mathrm{Cu}$ and the values of the parameters $\lambda_{d}, \Delta, \eta_{1}$ already used for skew scattering, is compared in Fig.1 with the skew scattering one. For impurities at the beginning and the end of the $5 d$ series $(L u, H f, I r, P t)$ the side jump contribution at $c=2 \%$ is much smaller than the skew scattering one. It is expected to remain smaller even at concentrations around 10\%. This is in agreement with the results of a constant SHA up to $c=12 \%$ for Ir in $C u$ [13]. On the other hand, for impurities in the middle of the series, like $W, T a$ or Os, concentrations as small as $2 \%$ yield side jump and skew scattering contributions of the same order of magnitude. For these impurities, very large contributions from the side-jump $\left(\Phi_{H}^{\text {anom }} \gtrsim 10 \%\right)$ are expected for concentrations of the order of $10 \%$. This can be compared to the situation of the Anomalous Hall Effect of $G d$ doped with $L u$ impurities [22] in which the side-jump contribution exceeds the skew scattering for concentrations above about $6 \%$ of $L u$.

The above discussion, for both the skew scattering and side-jump contributions, is altered when a crystal field splits the $t_{2 g}$ and $e_{g}$ states. According to the results summarized after Eq. (13), this introduces a change of sign not in the middle of the $5 d$ series but at midway through the filling the $t_{2 g}$ states at $Z_{t_{2 g}}=3$, and a reduction by 3 and 5 in the amplitudes of the Hall angles. This can change the variation through the $5 d$ series but not really the order of magnitude of the SHA's. For quantitative predictions only an $a b$-initio calculation of the scattering phase shifts can lead to realistic results. Our analytical calculation rather aims to predict the main features of what can be expected from $5 d$ resonances and to identify the important parameters.

Summarizing, large SHE effects induced by the resonant scattering from impurity states, here $d$ levels, are expected from the combination of: $i$ ) a large S-0 coupling of the impurity states and a narrow resonance, which is the condition to obtain a large asymmetric scattering amplitude in the resonance channel $l$, and $i i)$ a large symmetric scattering in the channels $(l \pm$ 1). The second condition is not really fulfilled for $5 d$ impurities in $C u$ so that skew scattering SHA's of only a few percent are expected in agreement with the existing experimental results. However, at least for some impurities $(W, T a, O s)$, large side jump effects can be expected 
with $\Phi_{H}$ exceeding $10 \%$ for $c \gtrsim 10 \%$. We anticipate that similar features can also be found for impurities with $p$ state resonances like $\mathrm{Pb}$ or $\mathrm{Bi}$. Spin Hall angles above $10 \%$ would be extremely interesting candidates for generating spin currents without magnetic materials in spintronic devices.

We thank Professor Elie Belorizky for helpful discussions on the projection of the scattering on crystal field states.

[1] M. I.Dyakonov and V. I. Perel, Phys. Lett. A 35, 459 (1971).

[2] R. Karplus and J. M. Luttinger, Phys. Rev. 95, 1154 (1954).

[3] J. Sinova, D. Culcer, Q. Niu, N. A. Sinitsyn, T. Jungwirth, A. H. MacDonald, Phys. Rev. Lett. 92, 126603 (2004).

[4] J. Smit, Physica (Amsterdam) 21, 877 (1955).

[5] L. Berger, Phys. Rev. B 2, 4559 (1970).

[6] T. Kimura, Y. Otani, T. Sato, S. Takahashi, S. Maekawa, Phys. Rev. Lett. 98, 156601 (2007) ; for a correct value of the SHA, see Erratum by T. Kimura, Y. Otani, T. Sato, S. Takahashi, S. Maekawa, Phys. Rev. Lett. 98, 249901(E) (2007). Note that the same group has recently announced a much larger value of the SHA in Pt, 1.4\%, see Ref.23.

[7] O. Mosendz, J.E. Pearson, F.Y. Fradin, G.E.W. Bauer, S.D. Bader, A. Hoffmann, Phys. Rev. Lett. 104, 046601 (2010).

[8] T. Seki, Y. Hasegawa, S. Mitani, S. Takahashi, H. Imamura, S. Maekawa, J. Nitta, K. Takanashi, Nature Mater. 7, 125 (2008).

[9] G. Y. Guo, S. Maekawa, N. Nagaosa, Phys. Rev. Lett. 102, 036401 (2009).

[10] K. Takanashi, et al., private communication.

[11] A. Fert, A. Friederich and A. Hamzic, J Magn. Magn. Mat. 24, 231 (1981).

[12] The definition of $\Phi_{H}$ by Fert et al., see Ref. [11], differs from the definition in current use today by a factor of $2\left(\Phi_{H}\right.$ was defined as the ratio of $\rho_{x y}$ to the global resistivity $=\frac{1}{2} \rho_{x x}$ ), so that the values of $\Phi_{H}$ in Table 3 of Ref.[11] have to be divided by 2 to be compared with the results of recent experiments and the calculations of this paper.

[13] Y. Niimi et al, abstract of the 2010 Conference on Magnetism and Magnetic Materials.

[14] Martin Gradhand, Dmitry V. Fedorov, Peter Zahn, and Ingrid Mertig, Phys.Rev.Lett. 104, 
186403 (2010); Phys.Rev.B 81,245109 (2010).

[15] E. Daniel, J. Friedel, Proc. Intern. Conf. On Low Temperature Physics, eds. J. Daunt, P. Edwards, F. Milford and M. Yaqub (Plenum, New York, 1965) p. 933.

[16] P.M. Levy, Phys. Rev. B38, 6779 (1988).

[17] See Ref.16, Sec. IV. Note that while a magnetic field is used in Ref.16 for the Hall effect, here we use a spin polarized current in which $\hat{z}$ denotes the axis of spin polarization.

[18] See Ref.16, Eq. 4.7.

[19] S. Hufner, G. K. Wertheim, J. H. Wernick, Solid State Com. 17, 1585 (1975).

[20] M. Chshiev et al., private communication.

[21] J.S. Griffith, The Theory of Transition-Metal Ions (Cambridge University Press, Cambridge, England 1961); see Secs. 5.1 and 5.2, and Fig. 5.1.

[22] R.Asomoza, A. Fert and R. Reich, J. Less Common Metals 90, 177 (1983).

[23] M. Morota et al., arXiv:sbmitt/0085533. 